\title{
15
}

\section{A Queueing Network Model of Distributed Shared Memory}

\author{
D. Venkatesulu* \& T.A. Gonsalves \\ Department of Computer Science and Engineering, \\ Indian Institute of Technology, \\ Madras 600036, India. \\ E-mail:dvn\%kurinji@iitm.ernet.in \\ tag@iitm.ernet.in
}

\begin{abstract}
Distributed Shared Memory (DSM) is a mechanism which provides logical shared memory in a distributed system. This simplifies the task of distributed programming. We develop simple queueing network models that can be used to compare the performance characteristics of several DSM algorithms for location of shared pages. Our models can be evaluated efficiently using the MVA (Mean Value Analysis) technique. Using these models, we can explore the regions of applicability of various DSM algorithms. By modelling contention at shared resources such as the network and memory servers, our model is able to provide more accurate results than the earlier analysis by Stumm and Zhou.
\end{abstract}

\section{Keywords}

Distributed Shared Memory, Computer Networks, Inter-Process Communication, Queueing Network Model, Mean Value Analysis.

\section{INTRODUCTION}

In a distributed system each node has its own processor and memory. Memory is not physically shared between any nodes. Traditionally, the I/O-based communication paradigm using message-passing is used in distributed programming. This is significantly more complex than the shared memory communication paradigm used in sharedmemory multiprocessors. Distributed Shared Memory (DSM) [Cheriton (1986), Li (1986), Fleisch (1988), Kessler et al. (1989), Stumm et al. (1990)] is a mechanism which provides an abstraction of logically shared memory on the physically distributed memory of a distributed system. By making the separation of memories transparent to the programmer, DSM supports data sharing directly and passing of complicated data structures by reference, and simplifies process migration. Several issues need to be considered in a DSM. These include [Nitzberg et al. (1991)]:

* Supported by a Govt. of India Research Scholarship. 


\section{- Structure and granularity of Memory}

Structure refers to the layout of the shared data in memory, it could be a variable length segment (object) or fixed length linear array of words, and Granularity refers to the size of the unit of sharing: byte, word, page, or complex data structure.

- Algorithms for Location of Shared Memory

There are several ways of locating shared data [Li (1986), Fleisch (1989)]. First one is the Centralized Manager which holds the information about the location of shared data at one place. The second one is the Broadcast of every request, which broadcasts every request to know the location of shared data. The third one is the Distributed Manager which reduces the load at the centralized manager and every node holds approximate information about the location of shared data.

\section{- Coherence Semantics and Protocols}

The most appealing semantics for memory coherence is the strict consistency. By strict consistency we mean that a read operation to a memory location should return most recently written value into that location. There are several algorithms to provide strict memory coherence [Cheriton (1986), Li (1986), Fleisch (1988), Kessler et al. (1989), Stumm et al. (1990)].

While DSM can be implemented in hardware, Memnet, [Delp (1988)], the use of software mechanisms akin to virtual memory is more common. Our interest is in such software mechanisms since these can be implemented on commercially available computers. Our basic objective is the efficient comparative evaluation of the performance of various DSM algorithms. The most promising approach is the use of approximate queueing network models which can be evaluated efficiently.

In the next section, we describe the various DSM algorithms that we study. Section 3 explains the workload characterization, System characteristics, queueing network models for these algorithms, Stumm \& Zhou's performance model, and validation of our model. Performance results and conclusions are presented in sections 4 and 5 respectively.

\section{DSM ALGORITHMS}

Stumm and Zhou have proposed four algorithms for data access in a DSM [Stumm et al. (1990)]. Of these, two are non-migratory and two are migratory. The latter algorithms migrate a shared data page from the owner node to the requesting node on demand, whereas the former hold shared data statically at each node.

The Central Server algorithm keeps only one copy of shared data in the system. The Migration algorithm allows only one read-write copy of a page to exist in the system at a time and migrates the page on demand to the requesting node. The Read-replication algorithm allows either one read-write copy or multiple read-only copies of a page to exist at a time. All read-only copies of a page are invalidated on a write to the page. Thus it is a write-invalidate algorithm. The Full-replication algorithm maintains readonly copies of each shared page at all nodes. It updates all copies of a page on a write operation, i.e it is a write-broadcast algorithm.

\subsection{Central Server Algorithm}

The simplest DSM algorithm has a single memory server that holds the entire shared memory of the system. Hence, every access to the shared data from client processes is remote which requires network access. Client processes on other nodes send requests 
explicitly to the server for every read or write of shared data. Each node also has local memory. The server serializes all the remote memory access requests ensuring strict consistency. However, there is no concurrency in processing the shared data access requests.

The advantage of this algorithm is that it is easy to implement and it is quite useful in client-server model applications. The disadvantage of this algorithm is that the central server will become the bottleneck of the system as the number of nodes increases.

One way of reducing the load at the central server is to distribute the shared data statically among several servers. The resultant increase in concurrency in processing shared memory requests will reduce the average waiting time. In the extreme case, every node acts as both a memory server and runs client processes. This could slow down the client processes.

\subsection{Migration Algorithm}

When a node references a page, it is likely to make additional references to it in the near feature. We can exploit this locality of reference by migrating the page to the requesting node. This node now becomes the new owner of the page. Initially the owner of a page will be the node at which it has been created. It is necessary to keep track of the location of pages. This can be performed by having a separate manager process running on one node which keeps track of the current owners of every page. The manager forwards the request to the owner of the page. Alternatively, the request could be broadcast.

The migration algorithm allows only one read/write copy of a page to exist at a time. The local copy of a page is invalidated when it is migrated to another node. The advantage of this algorithm is that it is similar to the virtual memory management system. It performs well when application exhibits a high degree of locality in its shared data accesses. It performs very poorly when there are interleaved read accesses to the same page by different nodes. This causes a page transfer between the two nodes for every access which increases the page fault rate.

The size of the page to be transferred also plays major role in determining the performance of this algorithm. As the size of the page increases, the transmission time at the network increases but the page fault rate could decrease.

\subsection{Read-replication Algorithm}

If a page has many readers with only occasional writes, the migration algorithm will experience considerable overhead as the page moves back and forth between readers. In such cases, the Read-replication algorithm can be advantageous. This allows either multiple read-only copies of a page or only one read-write copy of the page to be present in the system at a time. It solves the problem of memory coherence by invalidating all the read-only copies of a page on a write access to that page. The new owner of a page is the node at which the write fault occurred most recently. The owner holds either a read-write or a read-only copy of a page, and it maintains a list of nodes having readonly copies of a page, i.e the copy set. The owner of a page changes only on write faults. The manager keeps track of the owners of different pages.

On a read fault, the requesting node sends a message to the manager, which forwards the request to the owner. The owner adds the faulting node to the copy-set of the page, it then transfers a read-only copy of the page to the requesting node and changes its own access rights to read-only, if necessary. 
A write fault is similar to the read fault except that the owner transfers ownership and the copy-set of the page to the faulting node. The faulting node sends invalidation requests to the copy-set members. The faulting node will resume its write operation only after sending invalidation messages.

\subsection{Full-replication Algorithm}

To further reduce overhead when reads are predominant and there is a high degree of contention for a page between readers and writers, the Full-replication algorithm is more suitable. It maintains read-only copies of all pages at all nodes. On a write fault, the faulting node broadcasts the modification to all other nodes.

For consistency, the broadcast must be reliable and writes should be processed at all nodes in the same order. This can be achieved via a reliable broadcast protocol that uses one sequencer node. Each write operation is sent to the sequencer node which adds a sequence number to the request. It then broadcasts the request. It also maintains a history of recently received requests to handle retransmission requests. The sequence number of the request allow the server process at each node to determine whether the update request is a duplicate or whether it has missed any broadcasts. In the latter case, it requests retransmission from the sequencer.

\section{SYSTEM MODEL}

We now develop an approximate queueing network model to evaluate the performance of the DSM algorithms described in the previous section. First, we characterize the workload of a DSM system. Next, we present our model. Finally,we describe an earlier model [Stumm et al. (1990)].

\subsection{Workload Characterization}

The performance of DSM depends on the memory access behaviour of the processes running on the various nodes. We assume that a single process runs on each node. The memory accesses of the process are divided into two types: local access which does not cause a fault, and remote access which causes a fault. Local access includes all accesses to private memory, and accesses to shared memory that is locally resident. Only access to shared memory that is resident on another node such that a read or write fault is generated, is remote access.

Thus, a program is characterized by the lengths of the local burst, $Z$, between successive remote accesses [kessler et al. (1989)]. This is a random variable that is a complex function of both the interaction between different processes and also the DSM algorithm. Consider, for example, a series of accesses to the same shared page. In the Central server algorithm, every one will be a remote access. In the migration algorithm, on the first fault, the page is migrated and hence the remaining accesses become local ones. Thus, $Z_{\text {mig }}$ is expected to be greater than $Z c s$.

In algorithms with multiple read-only copies of a page, the read and write accesses may have different effects. Hence, the read-to-write ratio, $r$, is an important characteristic. For most programs, $r>1$. 


\subsection{System Characteristics}

The system consists of $N$ nodes interconnected by a broadcast network of capacity $C$. Each node has some private memory and some shared memory. We assume that there are two sizes of packets: short packets, typically about 64 bytes, are used for control, requests and individual data objects; large packets each contain a memory page (a large packet may actually consists of several maximum size LAN frames back-to-back). The processing cost of sending or receiving a small packet is $p$ and of a large packet is $P$.

Requests for shared memory access received from remote nodes are given higher priority than local processing. In addition to the $N$ nodes, some algorithms require a manager and/or sequencer. These are dedicated nodes that do not participate in user computation or hold shared memory. The service demand of each request at the manager and sequencer are denoted by $D \quad m$ and $D_{s e q}$ respectively.

\subsection{Queueing Network Model}

We have chosen analytical modelling to evaluate the different DSM algorithms, because it is less expensive than measurement or simulation and provides quick insight into the performance of a system. In order to efficiently evaluate models with large numbers of nodes, we resort to approximate mean value analysis (MVA) [Lazowska et al. (1984)]. We validate our models by comparison with published results (section 3.5).

Trace-driven simulation or measurement with actual distributed applications will certainly yield more accurate results than analytical models. However, these results would be applicable to only specific workloads. Distributed workloads are highly variable in their memory reference behaviour and communication patterns. Hence, the results of simulation or measurement may not yield insight into the system.

The workloads in our models do not correspond to any particular application. However, by focussing on relative performance of different algorithms for a wide range of parameters, we are able to obtain insights into DSM behaviour that could not be easily obtained otherwise. We do not, in general, ascribe especial importance to the absolute values of performance predictions.

We use closed network of queues to represent the system [Lazowska et al. (1984)]. Servers correspond to nodes, managers, sequencers and the network. Local access bursts and local packet processing are modelled by think time using the interactive workload. When the same node serves both local processing and remote requests, the remote requests get absolute priority. To model this, we use the technique of service demand inflation [Lazowska et al. (1984), Chap. 11]. We first find the utilization of the server, $U_{r}$, due to remote requests. The local processing time, $Z$, is then inflated to $Z^{\prime}=$ $1 /\left(1-U_{r}\right)$.

\subsubsection{Central Server Model}

We assume that the shared data is statically distributed among all $N$ nodes. That is, each node acts as a client and also as a memory server. Accesses to shared data are uniformly distributed. Hence, the probability that a shared access results in a fault is $(N-1) / N$. Each remote access takes two short packets. The access cost is $2 p$ at the requesting node and $2 p+$ memory access time at the memory server.

In our queueing network model for the Central server algorithm, Figure 1, each node is represented by two servers: a delay service centre to represent the client functions, and a queueing service centre that represents the memory server function. The network is represented by a queueing service centre. 


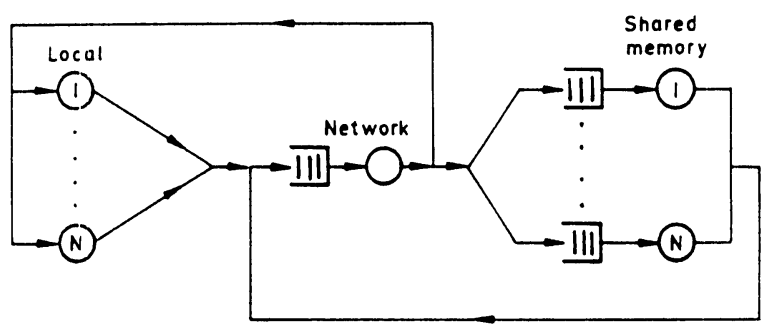

Figure 1 Model of Central server algorithm. Node $i$ is represented by local server $i$ and shared memory server $i$.

Each job corresponds to a local access burst followed by one remote access. Hence, we have the following service demands:

Local server, $l \quad$ local burst + two short packet events. $D_{l}=Z+2 p$

Memory server, $k \quad$ One memory access + two short packet events, multiplied by visit ratio, $1 / N . \quad D_{k}=(d+2 p) / N$, where $d$ is the local memory access time.

Network server, $n \quad$ Two short packet transmissions. $D_{n}=2 l / C$, where $l$ is the length of a short packet.

This allows concurrency between client and memory server processing at each node that does not exist in reality. To account for this, since by assumption the memory server runs at higher priority, we use demand inflation at the local server: $D_{l}^{\prime}=D_{l} /\left(1-U_{k}\right)$, where $U_{k}$ is the utilization of the memory server. Initially, $D_{l}^{\prime}$ is set to $D_{l}$ and we iterate until convergence. Finally, the average shared memory access time, $T_{C S}=(N-1) / N^{*}$ average remote access time.

\subsubsection{Migration Model}

To extend the Central server model to migration, we add a manager service centre which is a queuing centre with service demand $D_{m}$. Next, the service demands at the other centres are adjusted to account for the cost of one request packet(small) plus one data packet(large) for each page fault. Hence, we have the following service demands:

Local server, $l \quad$ local burst + one short packet event + one large data packet event. $D_{l}=Z+p+P$

Memory server, $k$ one short packet event + one large data packet event, multiplied by visit ratio, $1 / N$. $D_{k}=(p+P) / N$.

Manager server, $m \quad$ two short packet events. $D_{m}=2 p$.

Network server, $n$ two short packet transmissions + one large data packet transmission. $D_{n}=(2 l+L) / C$, where $L$ is the length of the large data packet.

As above, servicing of incoming remote requests is given higher priority and hence local processing is subjected to inflation. To compute the average shared memory access time, we assume a page fault rate (with out replication), $f$, for references to shared memory. As discussed earlier, $f$ would typically be smaller than the corresponding rate in Central server algorithm and is a complex function of program behaviour and memory page size. The average shared memory access time, $T_{m}=f^{*}$ average remote access time. 


\subsubsection{Read-replication Model}

The model of this algorithm is same as the migration algorithm with one exception. In this algorithm, the service demands at the memory servers vary with the read/write ratio $r$, and the number of nodes having replicated copies of a page. This is due to the fact that on every write operation, all read-only copies in the system are invalidated. We have the following service demands:

Local server, $l \quad$ local burst + one short packet event + one large data packet event. $D_{l}=Z+p+P$

Memory server, $k$ one short packet event + one large data packet event + average cost of invalidation, multiplied by visit ratio, $1 / N$. $D_{k}=(p+P+(1 /(r+1)) * N p) / N$

Manger server, $m \quad$ two short packet events. $D_{m}=2 p$

Network server, $n$ three short packet transmissions + one large data packet transmission. $D_{n}=(3 l+L) / C$.

The average shared memory access time, $T_{r r}=f^{\prime} *$ average remote access time, where $f^{\prime}$ is the average fault rate with replication.

\subsubsection{Full-replication Model}

Since every node has read-only copies of all pages, read operations on shared data are local accesses. Every write operation to shared memory requires that the writer to send the update request in a short packet to the sequencer. The sequencer then broadcasts the packet which is received and processed by all other nodes. Hence, we have the following service demands:

Local server, $l$

Memory server, $k$

Sequencer server, seq

Network server, $n$ local burst + one short packet event. $D_{F}=Z+p$.

one short packet event, multiplied by visit ratio, 1 . $D_{k}=p$.

two short packet events. $D_{\text {seq }}=2 p$.

two short packet transmissions. $D_{n}=2 l / C$.

Demand inflation and iteration is used as in the other models. The average shared memory access time, $T_{f r}=(1 /(r+1)) *$ average remote access time

\subsection{Stumm and Zhou's Model}

Stumm and Zhou have derived expressions for average access time using a simple deterministic model of the DSM algorithms [Stumm et al. (1990)]. They ignore network transmission time, local memory access time and queueing delays at servers. The average access time is given by the probability that an access to shared data is remote multiplied by the average remote access time. The first term depends on the number of nodes, $N$, in the case of Central server algorithm, on the fault rate, $f$ and $f^{\prime}$, in Migration and Read-replication, and read/write ratio, $r$, in Full-replication. The second term depends on the number of packets involved and the packet processing times. They have been derived expressions for the average access time:

Central Server $\quad T_{C S}=(1-1 / N) * 4 p$

Migration $\quad T_{m}=f^{*}(2 P+4 p)$ 


$$
\begin{array}{ll}
\text { Read-replication } & T_{r r}=f^{\prime} *(2 P+4 p+(1 /(r+1) * N p) \\
\text { Full-replication } & T_{f r}=(1 /(r+1)) *(N+2) p
\end{array}
$$

\subsection{Validation}

Since queueing network models are approximate models, it is necessary to validate these models by comparison with earlier models or with the results of measurement or simulation. First, we validate our model by comparing it with the results of Stumm \& Zhou. By setting the service demands to zero at the network and manager servers in the Migration model, and at the network server in Central server model, and by assuming the local processing time between successive remote requests is negligible, we are able to get the equal performing curve almost the same as Stumm \& Zhou's model as shown in Figure 2. Also in Figure 2, we have shown that the effect of increase in local processing time, when $D_{n}=0$ and $D_{m}=0 \mathrm{~ms}$.

We have also validated our model by comparing it with simulation results [Kessler et al. (1989)] for Hot Potato algorithm. We found that the difference is with in the acceptable limits (3-9\%).

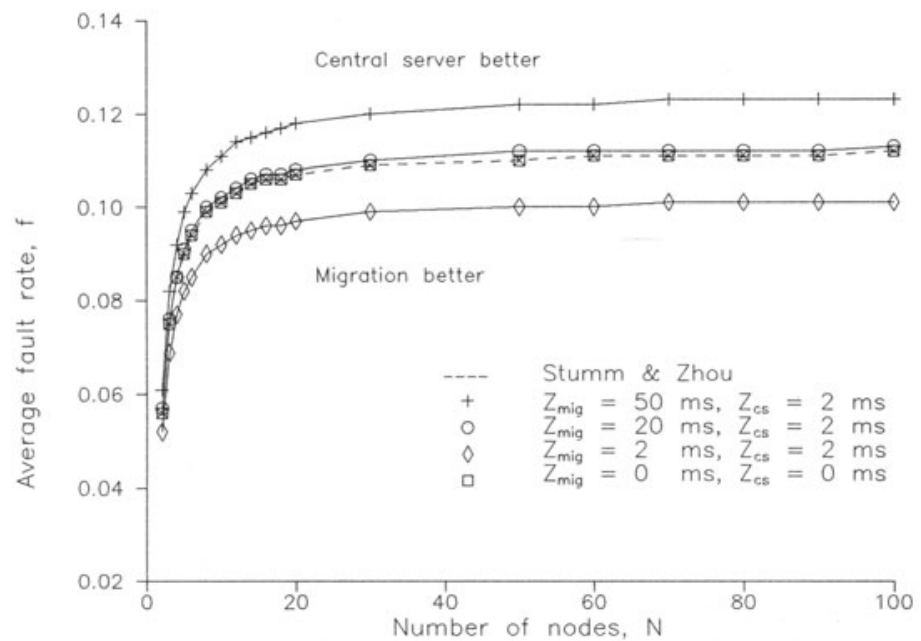

Figure 2 Validation of the Queueing Network Model

\section{PERFORMANCE RESULTS}

We now use our queueing network models to examine the trade-offs between the different DSM algorithms described in section 2. Since the memory reference behaviour of a distributed program is not well known, the absolute magnitudes of performance measures such as average memory access time are likely to be of limited use. However, the relative performance of different algorithms can be gauged. To this end, we present equal performing curves for each pair of algorithms. For two independent parameters, the average memory access time of both algorithms is the same along the equalperforming curve. Above the curve, one algorithm has higher performance, while below the other is better. 
Table 1 Parameter values used in the evaluations

Request/Response message size:

Memory page size:

Time to send/receive a message, $p$ :

Time to send/receive a Memory page, $P$ :

Network Bandwidth, $C$ :

Service demand at the manager, $D \mathrm{~m}$ :

Service demand at the Sequencer, $D$ seg

Local processing time, $Z$ :

Number of nodes, $N$ :
$64 \mathrm{~B}$

$8 \mathrm{~KB}$

$1 \mathrm{~ms}$

$16.75 \mathrm{~ms}$

$10 \mathrm{Mb} / \mathrm{s}-1 \mathrm{~Gb} / \mathrm{s}$

$0-2 \mathrm{~ms}$

$2 \mathrm{~ms}$

$0-100 \mathrm{~ms}$

$2-100$

We assume that the memory page size is $8 \mathrm{~KB}$ and that the CPU time to send or receive a request message (short packet), and a memory page (large data packet) is $1 \mathrm{~ms}$ and $16.75 \mathrm{~ms}$ respectively. We have assumed that the CPU is faster than the one assumed by Stumm \& Zhou. The network service demand is the message transmission time dependent on the network bandwidth. In the case of Central server and Fullreplication, one data item is contained in each request/response message. Allowing for overheads, these messages are assumed to be of size $64 \mathrm{~B}$. The local processing time between memory accesses in the central server algorithm, $Z c s$, is set to $2 \mathrm{~ms}$. In the case of the other algorithms, this varies depending on the locality of reference. Hence, $Z_{\text {mig }}$ is varied between 2 to $50 \mathrm{~ms}$. Table 1 is a list of the values used for each parameter.

\subsection{Central Server versus Migration}

To compare the Central server and Migration algorithms, for different number of nodes, $N$, we find the fault rate in the migration algorithm, $f$, at which the two algorithms perform equally. ${ }^{*}$ In Figure 3 equal-performing curves are plotted for $C=10,100$ $\mathrm{Mb} / \mathrm{s}, Z_{c s}=2 \mathrm{~ms}$ and $Z_{m i g}=2,20$ and $50 \mathrm{~ms} . Z_{m i g}=2 \mathrm{~ms}=Z_{c s}$ represents a program with very little locality of reference. $Z_{\text {mig }} \stackrel{m}{=} 20 \mathrm{~ms}$ and $50 \mathrm{~ms}\left(>>Z_{c S}\right)$ represent programs with greater locality of reference. For each case, migration performs better below the curve and central server is better above.

At Ethernet speeds, $10 \mathrm{Mb} / \mathrm{s}$, the central server algorithm performs better always. This is because the network is the bottleneck in the case of migration except for small $N$ when the contention for a page is reduced. For $N>40$, the degree of locality makes no difference. For $N<40$, performance of migration improves somewhat with higher locality (i.e $Z_{\text {mig }}=20$ and $50 \mathrm{~ms}$ ).

If the network bandwidth is increased to $100 \mathrm{Mb} / \mathrm{s}$, performance of migration improves further and we see that program locality has a larger impact except for large $N$. Further increase of $C$ to $1 \mathrm{~Gb} / \mathrm{s}$ does not improve performance (the curves overlap the $100 \mathrm{Mb} / \mathrm{s}$ ones and hence are not shown).

\footnotetext{
* Note that since we assume that all nodes act as servers, the fault rate in the central server algorithm is fixed at $(1-1 / \mathrm{N})$
} 
Also shown in Figure 3 is the curve using Stumm \& Zhou's analysis. Since they ignored the network overhead, and the queuing delay at the manager their model considerably over-estimates migration performance especially for large systems. For small $N$ (typically for $Z_{\text {mig }}=50 \mathrm{~ms}$ ), their model underestimates the performance of migration because they assumed that the local processing time between successive remote accesses is negligible.

In migration, every page fault requires service from the manager. Increasing the CPU speed of the manager node, i.e. reducing $D_{m}$ results in improvement of migration performance (Figure 4). At $10 \mathrm{Mb} / \mathrm{s}$, since the network is the bottleneck, a faster manager has minimal effect. At $100 \mathrm{Mb} / \mathrm{s}$, on the other hand, with $D_{m}<=1 \mathrm{~ms}$, performance improves substantially in large systems.

\section{Sensitivity Analysis}

Based on the equal performing curves, given expected system parameters, we can select the better algorithms. However, during operation, parameters are likely to vary by moving the system across the equal-performing curve to the region of poor performance for the selected algorithm. To see how serious this problem is likely to be, we have chosen the average remote access time for $N=50$, and the equal-performing point at $N$ $=50$, isdenoted by $f 50$. We now vary $f$ and plot average access time for both algorithms and various values of $C$ and $D \mathrm{~m}$.

At $C=10 \mathrm{Mb} / \mathrm{s}$, in Figure 5 there is a large difference between the two algorithms as we move away from equal performing point $f_{50}$ of $N=50$. For $C=100 \mathrm{Mb} / \mathrm{s}$, the difference decreases, becoming very small as $D_{m}$ decreases. That is, when the bottlenecks of network and manager are reduced, sensitivity to fault rate $f$ at $N=50$ is much less.

\subsection{Central server versus Full-replication}

Since Central server performs remarkably well relative to Migration, at low network bandwidths and large systems, we compare it to Full-replication. In Figure 6, we plot equal-performing curves with average read-to-write ratio, $r$, as the dependent variable. In each case, Full-replication is better above the curve and Central server is better below.

Central server performance is independent of $r$. As $N$ increases, $r$ must increase for Full-replication to remain equal to that of Central server. This is because the number of write faults increases with $N$. At $10 \mathrm{Mb} / \mathrm{s}$, for $N>70$, the network becomes the bottleneck in Central server and hence the curve levels off. At $C=100 \mathrm{Mb} / \mathrm{s}$, this does not happen even at $N=100$. Stumm \& Zhou's model consistently underestimates the average access time of full-replication. This because of the fact that they ignored the network overhead, and queueing delays at the sequencer and the memory servers. It overestimates the performance of full-replication only for large values of $Z_{f r}$ (typically $Z_{f r}=100 \mathrm{~ms}$ ). This is because of the fact that they have not taken into account the local processing time between successive requests. As in the previous section, we find that with the more queueing analysis, the region of applicability of the Central server algorithm is greater than predicted by the earlier analysis.

Up to $N=100$, we find that the utilization of the sequencer node is less than $100 \%$. Hence, a faster sequencer node will not affect performance except possibly in much larger systems. 


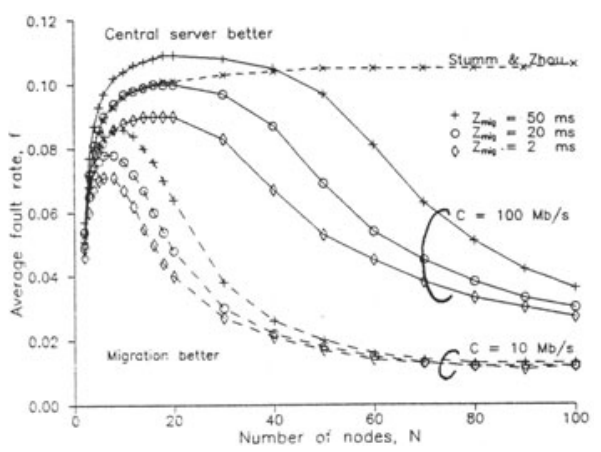

Figure 3 Equal-performing curves for Central server and Migration -- Central server is better above each curve, Migration is better below $\left(D_{m}=2 \mathrm{~ms}\right.$, $Z_{C S}=2 \mathrm{~ms}$ ).

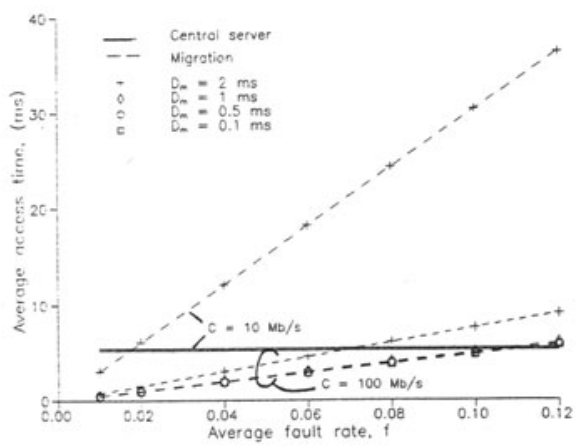

Figure 5 Sensitivity of equal-performing curves to change in average fault rate, $f$, Central server versus Migration. Page fault rate $f_{50}$, for Migration is the value that gives the equal-performing point for $N=50$.

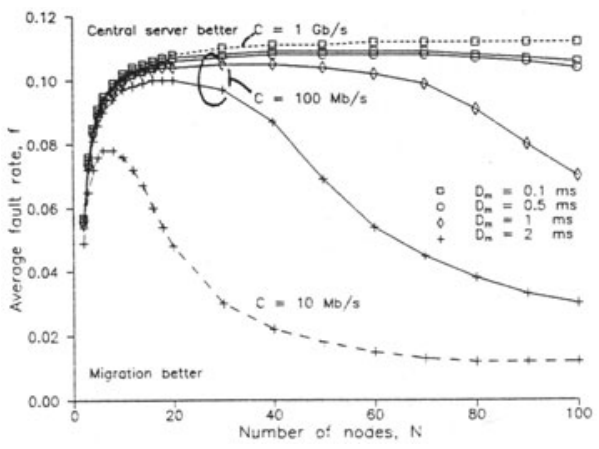

Figure 4 Effect of Manager service demand, $D_{m}$, on Central server-Migration equal-performing curves $\left(Z_{C s}\right.$ $=2 \mathrm{~ms}, Z_{\text {mig }}=20 \mathrm{~ms}$ ).

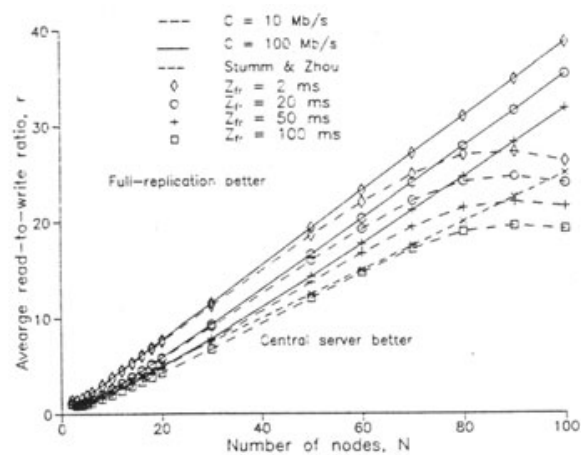

Figure 6 Equal-performing curves for Central server and Full-replication -Central server is better below each curve, Full-replication is better above $\left(D_{\text {seq }}=2\right.$ $\left.\mathrm{ms}, Z_{C S}=2 \mathrm{~ms}\right)$. 


\section{Sensitivity Analysis}

As in the previous section, in order to see the impact of moving the system across the equal-performing curve, we have chosen the average remote access time for $N=20,50$, and 80 for both the algorithms. The equal-performing point is denoted by $m 0$ for $N=$ 20. We now vary $r$ and plot average access time for both the algorithms for different values of $N$ and $C$. In Figure 7, we have chosen the $\mathrm{Y}$-axis in log scale.

The curves for $C=100 \mathrm{Mb} / \mathrm{s}$ for both the algorithms are overlapping with $C=10$ $\mathrm{Mb} / \mathrm{s}$ curves. Hence, we have not shown them in Figure 7 . For small $N$, the difference between the two algorithms as we move away from equal-performing point, $m_{20}$, is significant. The difference decreases, becoming small, as $N$ increases. Hence, for large $N$, sensitivity to large $r$ is small.

\subsection{Read-replication versus Full-replication}

We now compare Read-replication with Full-replication. In Figure 8, we plot equalperforming curves for various parameter values. At a low data rate of $C=10 \mathrm{Mb} / \mathrm{s}$, full-replication is almost always better than read-replication. In the latter, the network is the bottleneck as the entire memory page must be transferred to each reader after a write fault. In full-replication, only the relatively small data item is sent to all the readers. That is the network cost of a write fault in read-replication is higher than in full-replication.

At high values of read/write ratio, $r$, in Figure 8, full-replication does better comparatively because it always maintains up to date read-only copies at all nodes thus reducing the number of read faults. For low $r$, frequent updating is counter-productive and read-replication performance improves.

As expected, at $10 \mathrm{Mb} / \mathrm{s}$, in Figure $8 \mathrm{Stumm} \mathrm{\&} \mathrm{Zhou's} \mathrm{model} \mathrm{considerably} \mathrm{over-}$ estimates read-replication performance because it ignores the network overhead. At higher bandwidths, the correspondence is a bit better. However, there is still considerable inaccuracy because of the other effects such as the manager.

With $C=100 \mathrm{Mb} / \mathrm{s}$, in Figure 9, read-replication is seem to perform much better. For $N>50$, the curve levels off due to the manager node in read-replication. If a faster $\mathrm{CPU}$ is used in the manager, we see that read-replication performs even better. As we increase the speed of the CPU at the manager the effect of $D \quad m<0.5 \mathrm{~ms}$ is minimal.

\section{CONCLUSIONS}

We have developed an efficient approximate queueing network model of several DSM algorithms. By modelling shared resources such as the network and the manager, and by considering queueing delays, these models are substantially more accurate than earlier ones.

Our results show that the Central server algorithm does surprisingly well compared to migration algorithm. This is especially true at low network data rates of $10 \mathrm{Mb} / \mathrm{s}$. Full-replication performs well, especially at high read/write ratios.

To improve confidence in these models, we plan to perform more extensive validation by comparison with simulation. We are currently using our model to predict the performance of a DSM-Based Distributed Programming Testbed [Hariram \& Gonsalves 94], and are extending it to hybrid algorithms. 


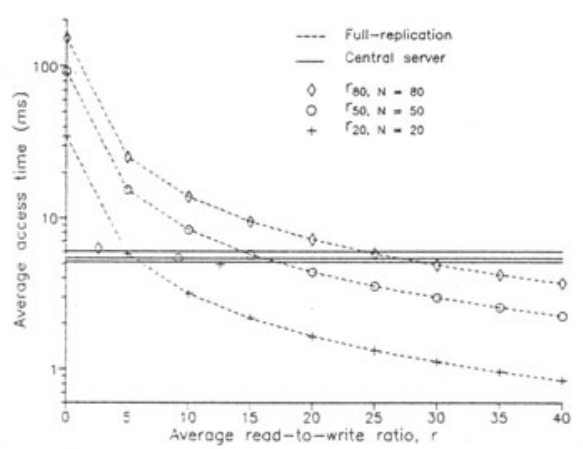

Figure 7 Sensitivity of equal-performing curves to change in read/write ratio, $r$, Central server versus Full-replication. Write fault rate 20 , for Full-replication is the value that gives the equal-performing point for $N=20\left(Z_{C S}=2 \mathrm{~ms}, Z_{f r}=20\right.$, and $N=80,50$, and 20).

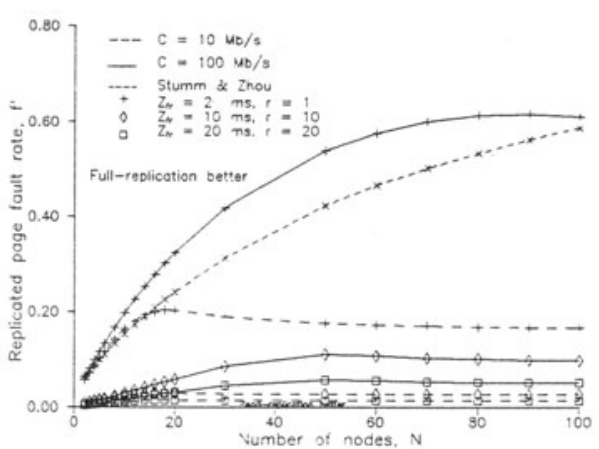

Figure 8 Equal-performing curves for Read-replication and Full-replication -read-replication is better below each curve and full-replication is better above $\left(D_{m}=2 \mathrm{~ms}\right.$, and $\left.Z_{r r}=20 \mathrm{~ms}\right)$.

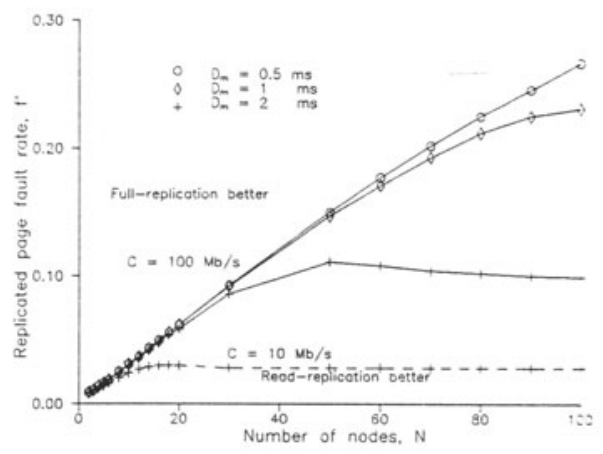

Figure 9 Effect of manager service demand, $D_{m}$, on Read-replication - Fullreplication equal-performing curves $\left(Z f r=10 \mathrm{~ms}, Z_{r r}=20 \mathrm{~ms}\right.$, and $\left.r=10\right)$.

\section{REFERENCES}

Agarwal, A. and Gupta, A.(1988) Memory Reference Characteristics of Multiprocessor Applications Under MACH. Proc. ACM SIGMETRICS '88 Conf. on Meas. and Modelling of Comp. Sys., 16, 1, 88, pp. 215-225.

Archibald, J. and Baer, J.(1986) An Evaluation of Cache Coherence Solutions in Shared-Bus Multiprocessors. ACM Trans. Comp. Sys., Vol. 4, No. 4, Nov. 86, pp. 273-298. 
Bennet, J. Carter, J. and Zwaenepoel, W.(1990) Munin: Distributed Shared Memory Based on Type-Specific Memory Coherence. Proc. 90 Conf. Principles and Practice of Parallel Programming, ACM Press, New York, N.J., 90, pp. 168-176.

Cheriton, D.R.(1986) Problem-Oriented Shared Memory: A Decentralized Approach to Distributed System Design. Proc. $6^{\text {th }}$ Int'l Conf. Distributed Computing Sys., May 86, pp. 190-197.

Delp, G.S.(1988) The Architecture and Implementation of Memnet: A High-Speed Shared Memory Computer Communication Network. Ph.D thesis, Univ. of Delaware, Newark, Del., 88.

Denning, P.J. and Buzen, J.P.(1978) The Operational Analysis of Queueing Network Models. ACM Computing Surveys, Vol. 10, No., Sep. 78, pp. 225-261.

Dubois, M and Wang, J.C.(1991) Shared Block Contention in a Cache Coherence Protocol. IEEE Trans. on Computers, Vol. 40, No. 5, May 91, pp. 640-644.

Eggers, S.J.(1991) Simplicity Versus Accuracy in a Model of Cache Coherency Overhead. IEEE Trans. on Computers, Vol. 40, No. 8, Aug. 91, pp. 893-906.

Fleisch, B.D.(1987) Distributed Shared Memory in a Loosely Coupled Distributed System. Proc. of the ACM SIGCOMM' 87 Workshop, Stowe, Vermont, Aug. 87, pp. 317-327.

Fleisch, B.D. and Popek, G.J.(1989) Mirage: A Coherent Distributed Shared Memory Design. Proc. $12^{\text {th }}$ ACM Symp. Op. Sys. Prin., Dec. 89, pp. 211-222.

Hariram, R.K. and Gonsalves, T.A.(1994) A DSM-Based Portable Distributed Programming Testbed. Proc. NETWORKS '94: An Int'l Conference on Comp. Net., Arch. and Appln., Madras, India, Dec. 94.

Kessler, R.E. and Livny, M.(1989) An Analysis of Distributed Shared Memory Algorithms. Proc. $9^{\text {th }}$ Int'l Conf. Distributed Computing Sys., CS Press, Los Alamitos, Calif., June 89, pp. 498-505.

Lazowska, E.D., Zahorjan, J., Graham, J.S. and Seivick, K.C.(1984) Quantitative System Performance-Computer System Analysis Using Queueing Network Models. Prentice-Hall Inc., Englewood Cliffs, NJ, 84.

Li, K. and Hudak, P.(1986) Memory Coherence in Shared Virtual Memory Systems. ACM Trans. Computing Sys., Vol.7, No. 4, Nov. 89, pp. 229-243.

Nitzberg, B. and Lo, V.(1991) Distributed Shared Memory: A Survey of Issues and Algorithms. IEEE Computer, Aug. 91, pp. 52-60.

Ramachandran, U. and Khalidi, M.Y.A.(1991) An Implementation of Distributed Shared Memory. Software-Practice and Experience, Vol.21, No. 5, May 91, pp. 443-464.

Stumm, M. and Zhou, S.(1990) Algorithms Implementing Distributed Shared Memory. IEEE Computer, Vol. 23, No. 5, May 90, pp. 54-64.

Zhou, S., Stumm, M. and McInerney, T.(1990) Extending Distributed Shared Memory to Heterogeneous Environments. Proc. $10^{\text {th }}$ Int'l Conf. Distributed Computing Sys., Los Alamitos, Calif, May-June 90, pp. 30-37.

Zhou, S., Stumm, M., Li, K. and Wortman, D.(1992) Heterogeneous Distributed Shared Memory. IEEE Trans. on Parallel and Distributed Systems, Vol. 3, No. 5, Sep. 92, pp. 540-554. 


\section{BIOGRAPHY}

D. Venkatesulu received his M.Sc. degree in Physics and M.Tech degree in Computer Science in 1985 and 1988 from Andhra University. He was in the CSE Faculty of Nagarjuna University from 1988 to 1990 . He is currently pursuing his Ph.D degree at IIT Madras. His research interests include distributed systems, performance evaluation, and computer networks.

Timothy A. Gonsalves received a B.Tech.(Electronics) from I.I.T.,Madras in 1976, and M.S. (EE) from Rice University, Houston, USA in 1978, and a Ph.D.(EE) from Stanford University, USA in 1986.

From 1986 to 1988 he was an Assistant Professor in the Computer Science Department, Worcester Polytechnic Institute, USA. In January 1989, he joined the Department of Computer Science and Engineering, I.I.T.,Madras where he is currently an Associate Professor. His research interests include distributed computer systems, computer networks, and performance evaluation. He is active in technology/product development and consultancy for a number of industries. Dr. Gonsalves is a member of CSI, ACM and IEEE. 